\title{
Geodesic diameter of sets defined by few quadratic equations and inequalities
}

\author{
Michel Coste and Seydou Moussa
}

November 2, 2018

\begin{abstract}
We prove a bound for the geodesic diameter of a subset of the unit ball in $\mathbb{R}^{n}$ described by a fixed number of quadratic equations and inequalities, which is polynomial in $n$, whereas the known bound for general degree is exponential in $n$. Our proof uses methods borrowed from D'Acunto and Kurdyka (to deal with the geodesic diameter) and from Barvinok (to take advantage of the quadratic nature).

Mathematics Subject Classification: 14P10
\end{abstract}

\section{Introduction}

Quantitative bounds on the topology or the geometry of semialgebraic sets are often given in terms of the number $k$ of polynomials used to describe them, the maximal degree $d$ of these polynomials and the number $n$ of variables. As a general rule, these bounds are exponential in the number of variables. One of the most famous bounds on the topology is the Petrovskii-Oleinik-Thom-Milnor bound $d(2 d-1)^{n-1}$ on the sum of the Betti numbers of a real algebraic sets (this bound and many others can be found in [7]). An interesting example of a bound concerning the geometry is the one given by D'Acunto and Kurdyka on the geodesic diameter of (a bounded part of) a real algebraic or semialgebraic set, which is of the form $O(d)^{n-1}([9,10,14])$.

Semialgebraic sets defined by quadratic polynomials do have a specific behaviour, in contrast with the general exponential bounds. The first result in this direction was obtained by Barvinok [2, who proved a polynomial bound of type $n^{0(k)}$ for the sum of the Betti numbers of subsets of $\mathbb{R}^{n}$ defined by a fixed number $k$ of quadratic inequalities. There are now several papers (for instance [12, 3, 4, 15, 6]) with bounds polynomial in the number of variables concerning the quadratic case.

In the present paper we show the same polynomial (in the number $n$ of variables) behaviour for a metric invariant: our main result is a bound of type $n^{O(k)}$ for the geodesic diameter of (a bounded part of) a semialgebraic subset of $\mathbb{R}^{n}$ defined by $k$ quadratic equations and inequalities. Section 2 of the paper establishes the result in the key case of a smooth complete intersection of quadrics. In order to treat this case, we extend and apply the methods of D'Acunto and Kurdyka on the one hand and of Barvinok on the other hand. 
Specifically, we use the idea of D'Acunto and Kurdyka of controlling the geodesic diameter by the length of trajectories of the gradient of a Morse function, which is in turn bounded by the length of the "thalweg" of this function the locus of points where the level sets are the most far apart - which has the advantage of being semialgebraic. A tricky point is to show that this thalweg can be assumed of dimension 1 . The complication with respect to the work of D'Acunto and Kurdyka comes from the fact that we have to deal with a complete intersection and cannot reduce to the hypersurface case, which would destroy the quadratic nature of the equations. The length of the thalweg is then estimated via Cauchy-Crofton formula, by counting intersection points with a hyperplane. This eventually leads to a system of equations containing a big (of size $n$ ) linear system in the variables $x \in \mathbb{R}^{n}$, with parameters including Lagrange multipliers, and a few $(O(k))$ other equations; the linearity in $x$ comes, of course, from the fact that gradients of quadratic functions are linear. Here we use Barvinok's method of solving the linear system in $x$ (as a function of the parameters and a few free variables among $x$ ) and carrying this solution in the other equations. The point is to show that the number of free variables, that is the corank of the linear system, can be assumed to remain small (controlled by $k$ ) when the parameters vary; this will give a bound polynomial in $n$ of degree $O(k)$ on the number of solutions of the complete system. The main difficulty here with respect to the situation considered by Barvinok is that the matrix of the system does not depend linearly on the parameters.

Section 3 of the paper shows how the general case can be reduced to the case of a smooth complete intersection of quadrics. We conclude with a few questions related to the fact that the bound obtained is surely far from optimal.

\section{Smooth complete intersection of quadrics}

We denote by $\mathbb{R}_{2}\left[X_{1}, \ldots, X_{n}\right]$ the space of polynomials of degree $\leq 2$. Let $Q_{i} \in \mathbb{R}_{2}\left[X_{1}, \ldots, X_{n}\right]$ for $i=1, \ldots, k$. Set

$$
X=\left\{x \in \mathbb{R}^{n} \mid Q_{1}(x)=\ldots=Q_{k}(x)=0\right\}, \quad M=X \cap \bar{B}^{n},
$$

where $\bar{B}^{n}$ denotes the closed unit ball (the open unit ball will be denoted by $\left.B^{n}\right)$.

We assume in this section that

(i) $M$ is a union of connected components of $X$, all contained in $B^{n}$, and a smooth complete intersection of codimension $k$ in $\mathbb{R}^{n}$.

In particular, the gradients $\nabla Q_{i}(x)$ are linearly independant at every point $x \in M$.

Our aim is to bound the sum of the geodesic diameters of the connected components $M_{i}$ of $M$. Recall that the geodesic distance between two points $x$ and $y$ in $M_{i}$ is the infimum of the lengths of paths joining $x$ to $y$ inside $M_{i}$, and that the geodesic diameter of $M_{i}$ is the supremum of the geodesic distances between two points of $M_{i}$. 


\subsection{The way by D'Acunto and Kurdyka: trajectories of gradient and thalweg}

Following D'Acunto and Kurdyka [9, 10, we shall use a Morse function on $M$. For every $M$ satisfying (i), it is possible to find $P \in \mathbb{R}_{2}\left[X_{1}, \ldots, X_{n}\right]$ such that

(ii) the restriction $\left.P\right|_{M}$ of $P$ to $M$ is a Morse function, and the critical values at the different critical points are distinct.

Observe that the set of $\left(P, Q_{1}, \ldots, Q_{k}\right)$ satisfying (i) and (ii) is a non empty open subset $\mathcal{U}$ of $\left(\mathbb{R}_{2}\left[X_{1}, \ldots, X_{n}\right]\right)^{k+1}$.

We denote by $\nabla_{M} P(x)$ the gradient of the restriction $\left.P\right|_{M}$ at a point $x \in M$. This is the orthogonal projection of $\nabla P(x)$ onto the tangent space $T_{x} M$, which is the orthogonal complement of the linear space spanned by the $\nabla Q_{i}(x)$ for $i=1, \ldots, k$. Hence, we have

$$
\nabla_{M} P(x)=\nabla P(x)-\sum_{i=1}^{k} u_{i}(x) \nabla Q_{i}(x),
$$

where

$$
G\left(\begin{array}{c}
u_{1} \\
\vdots \\
u_{k}
\end{array}\right)=\left(\begin{array}{c}
\left\langle\nabla Q_{1}, \nabla P\right\rangle \\
\vdots \\
\left\langle\nabla Q_{k}, \nabla P\right\rangle
\end{array}\right) \quad \text { and } \quad G=\left(\left\langle\nabla Q_{i}, \nabla Q_{j}\right\rangle\right)_{i, j=1, \ldots, k} .
$$

Here $\langle\cdot \mid \cdot\rangle$ denotes the standard scalar product on $\mathbb{R}^{n}$, and $\|\cdot\|$ will denote the standard euclidean norm.

A trajectory of the normalized gradient of $P$ on $M$ will be a continuous, piecewise smooth curve $x: I \rightarrow M$, where $I$ is an interval of $\mathbb{R}$, which satisfies $x^{\prime}(t)=\nabla P(x(t)) /\|\nabla P(x(t))\|$ for every $t \in I$ such that $\|\nabla P(x(t))\| \neq 0$.

The idea of D'Acunto and Kurdyka to bound the geodesic diameter of a connected component $M_{i}$ using trajectories of the normalized gradient is clear in the case when the Morse function $P$ has only one minimum and one maximum: two trajectories issued from points $x$ and $y$ in $M_{i}$ meet at the point where $P$ attains its maximum on $M_{i}$. Hence the geodesic diameter of $M_{i}$ is bounded by twice the maximal length of trajectories on $M_{i}$.

In order to bound the length of the trajectories of the normalized gradient, D'Acunto and Kurdyka use the "thalweg" of $P$ on $M$ : this is the set of points $x \in M$ such that $\left\|\nabla_{M} P\right\|$ has a local minimum at $x$ on the compact hypersurface $P^{-1}(P(x)) \cap M$. Roughly speaking, the thalweg is the place where the level hypersurfaces of $P$ on $M$ are the most far apart. The thalweg of $P$ on $M$ is contained in the set $\theta_{M}(P)$ of critical points of $\left\|\nabla_{M} P\right\|$ restricted to level sets of $\left.P\right|_{M}$. This is a semialgebraic subset of $M$.

Proposition 1 (D'Acunto-Kurdyka [10], Lemma 5.2) If $\theta_{M}(P)$ has dimension 1, then the sum of the geodesic diameters of the connected components of $M$ is bounded by twice the length of $\theta_{M}(P)$.

Our first task is to ensure that $\theta_{M}(P)$ has dimension 1, at least generically. Specifically we show 
Proposition 2 There is an open dense subset $\mathcal{U}^{\prime}$ of $\mathcal{U}$ such that, for every $\left(P, Q_{1}, \ldots, Q_{k}\right)$ in $\mathcal{U}^{\prime}$, the corresponding set $\theta_{M}(P)$ has dimension 1.

D'Acunto and Kurdyka show a similar result in their paper ([10, Proposition $4.5)$, but they consider only the case where $M$ is a hypersurface, which allows to proceed in a simpler way. Nevertheless, we shall follow the main lines of their proof.

The set $\theta_{M}(P)$ may be described, using Lagrange multipliers, as the set of points $x \in M$ such that there exist $\lambda, \mu_{1}, \ldots, \mu_{k}$ in $\mathbb{R}$ for which

$$
\nabla\left(\left\|\nabla_{M} P\right\|^{2}\right)(x)=\lambda \nabla P(x)+\sum_{i=1}^{k} \mu_{i} \nabla Q_{i}(x) .
$$

Using the formulas (11) and (2), and taking into account the fact that $\nabla_{M} P$ is orthogonal to each $\nabla Q_{i}$, we obtain

$$
\nabla\left(\left\|\nabla_{M} P\right\|^{2}\right)=2\left(\operatorname{He}(P)-\sum_{i=1}^{k} u_{i} \operatorname{He}\left(Q_{i}\right)\right) \nabla_{M} P,
$$

where $\operatorname{He}(P)$ (resp. $\left.\operatorname{He}\left(Q_{i}\right)\right)$ denotes the Hessian matrix of $P$ (resp. of $Q_{i}$ ).

For $x \in M$, we denote by $p_{x}$ the orthogonal projection on the tangent space $T_{x} M$.

Lemma 3 For $x \in M$, we have $x \in \theta_{M}(P)$ if and only if $\nabla_{M} P(x)$ is either null or an eigenvector of $p_{x} \circ\left(\operatorname{He}(P)(x)-\sum_{i=1}^{k} u_{i}(x) \operatorname{He}\left(Q_{i}\right)(x)\right)$.

Proof. Apply the projection $p_{x}$ to Equation (3), using (4).

We denote by $\operatorname{Sym}_{n}$ the space of symmetric real matrices of size $n$ and by $\mathbb{G}_{n-k, n}$ the Grassmannian of linear subspaces of $\mathbb{R}^{n}$ of dimension $n-k$. If $N \in \mathbb{G}_{n-k, n}$, we denote by $p_{N}$ the orthogonal projection onto $N$. Set

$$
\widehat{\Sigma}=\left\{(A, N, V, \lambda) \in \operatorname{Sym}_{n} \times \mathbb{G}_{n-k, n} \times \mathbb{R}^{n} \times \mathbb{R} \mid p_{N}\left(A\left(p_{N}(V)\right)\right)=\lambda p_{N}(V)\right\}
$$

and let $\Sigma \subset \operatorname{Sym}_{n} \times \mathbb{G}_{n-k, n} \times \mathbb{R}^{n}$ be the image of $\widehat{\Sigma}$ by the projection $(A, N, V, \lambda) \mapsto$ $(A, N, V)$. The set $\Sigma$ is interesting for us because, by Lemma 3, $\theta_{M}(P)$ is the set of $x \in M$ such that

$$
\left(\operatorname{He}(P)(x)-\sum_{i=1}^{k} u_{i}(x) \operatorname{He}\left(Q_{i}\right)(x), T_{x} M, \nabla P\right) \in \Sigma .
$$

Lemma 4 The semialgebraic set $\Sigma$ is a subset of codimension $n-k-1$ of $\operatorname{Sym}_{n} \times \mathbb{G}_{n-k, n} \times \mathbb{R}^{n}$

Proof. We begin by computing the dimension of the algebraic set $\varphi^{-1}(0)$, where

$$
\varphi: \operatorname{Sym}_{n-k} \times \mathbb{R}^{n-k} \times \mathbb{R} \longrightarrow \mathbb{R}^{n-k}
$$

is defined by $\varphi(B, W, \lambda)=\left(B-\lambda I_{n-k}\right) W$. The partial derivative $\partial \varphi / \partial B$ at $(B, W, \lambda)$ is the linear mapping $T \mapsto T W$ from $\operatorname{Sym}_{n-k}$ to $\mathbb{R}^{n-k}$. Hence, $\varphi$ is 
submersive at points $(B, W, \lambda)$ such that $W \neq 0$. It follows that the codimension of $\varphi^{-1}(0)$ in $\operatorname{Sym}_{n-k} \times \mathbb{R}^{n-k} \times \mathbb{R}$ is $n-k$.

Next we compute the codimension in $\operatorname{Sym}_{n} \times \mathbb{R}^{n} \times \mathbb{R}$ of

$$
\widehat{\Sigma}_{N}=\left\{(A, V, \lambda) \in \operatorname{Sym}_{n} \times \mathbb{R}^{n} \times \mathbb{R} \mid p_{N}\left(A\left(p_{N}(V)\right)\right)=\lambda p_{N}(V)\right\},
$$

for $N \in \mathbb{G}_{n-k, n}$. It is sufficient to do this for the subspace $N_{0}=\mathbb{R}^{n-k} \times\{0\}$; indeed, if $U$ is an orthogonal matrix carrying $N_{0}$ to $N$, then $\widehat{\Sigma}_{N}$ is the image of $\widehat{\Sigma}_{N_{0}}$ by the isomorphism $(A, V, \lambda) \mapsto\left(U A^{t} U, U V, \lambda\right)$. Now $\widehat{\Sigma}_{N_{0}}$ is the inverse image of $\varphi^{-1}(0)$ by the linear surjection $\operatorname{Sym}_{n} \times \mathbb{R}^{n} \times \mathbb{R} \rightarrow \operatorname{Sym}_{n-k} \times \mathbb{R}^{n-k} \times \mathbb{R}$ obtained by truncating symmetric matrices at their first $n-k$ rows and columns and vectors at their first $n-k$ coordinates. We conclude that the codimension of $\widehat{\Sigma}_{N}$ in $\operatorname{Sym}_{n} \times \mathbb{R}^{n} \times \mathbb{R}$ is always $n-k$, and consequently the codimension of $\widehat{\Sigma}$ in $\operatorname{Sym}_{n} \times \mathbb{G}_{n-k, n} \times \mathbb{R}^{n} \times \mathbb{R}$ is also $n-k$.

The set $\Sigma$ is the projection of $\widehat{\Sigma}$, and the restriction of this projection to the subset of $(A, N, V, \lambda)$ in $\widehat{\Sigma}$ such that $p_{N}(V) \neq 0$ is injective. It follows that $\Sigma$ has the same dimension as $\widehat{\Sigma}$, and this concludes the proof of the lemma.

We now use quadratic perturbations of $P$ and the $Q_{i}$ 's.

Fix $\left(P, Q_{1}, \ldots, Q_{k}\right)$ in $\mathcal{U}$ and consider a perturbation $\left(\widetilde{P}, \widetilde{Q}_{1}, \ldots, \widetilde{Q}_{k}\right)$ given by

$$
\left\{\begin{array}{rl}
\widetilde{P}(x) & =P(x)+\frac{1}{2}^{t} x H x+{ }^{t} L_{0} x \\
\widetilde{Q}_{i}(x) & =Q_{i}(x)+{ }^{t} L_{i} x+c_{i}, \quad i=1, \ldots, k
\end{array},\right.
$$

where $H \in \operatorname{Sym}_{n}, L=\left(L_{0}, L_{1}, \ldots, L_{k}\right) \in\left(\mathbb{R}^{n}\right)^{k+1}$ and $c=\left(c_{1}, \ldots, c_{k}\right) \in \mathbb{R}^{k}$. We can choose an open neighborhood $W$ of

$$
M=\left\{x \in \bar{B}^{n} \mid Q_{1}(x)=\ldots=Q_{k}(x)=0\right\}
$$

contained in $B^{n}$ and an open neighborhood $\mathcal{R}$ of the origin in $\operatorname{Sym}_{n} \times\left(\mathbb{R}^{n}\right)^{k+1} \times$ $\mathbb{R}^{k}$ such that, for every $(H, L, c) \in \mathcal{R}$,

- $\left(\widetilde{P}, \widetilde{Q}_{1}, \ldots, \widetilde{Q}_{k}\right)$ defined in (6) is in $\mathcal{U}$,

- $\widetilde{M}=\left\{y \in \bar{B}^{n} \mid \widetilde{Q}_{1}(y)=\ldots=\widetilde{Q}_{k}(y)=0\right\}$ is contained in $W$,

- for every $x \in W, \nabla \widetilde{Q}_{1}(x), \ldots, \nabla \widetilde{Q}_{k}(x)$ are linearly independant and, consequently, the matrix $\widetilde{G}(x)=\left(\left\langle\nabla \widetilde{Q}_{i}(x), \nabla \widetilde{Q}_{j}(x)\right\rangle\right)_{i, j=1, \ldots, k}$ is invertible.

We define

$$
\Psi: W \times \mathcal{R} \times \mathbb{R}^{k} \longrightarrow \operatorname{Sym}_{n} \times \mathbb{G}_{n-k, n} \times \mathbb{R}^{n} \times \mathbb{R}^{k} \times \mathbb{R}^{k}
$$

with components $\Psi_{1}, \ldots, \Psi_{5}$ by

$$
\begin{aligned}
\Psi_{1}(x,(H, L, c), u) & =\operatorname{He}(\widetilde{P})(x)-\sum_{i=1}^{k} u_{i} \operatorname{He}\left(\widetilde{Q}_{i}\right)(x), \\
\Psi_{2}(x,(H, L, c), u) & =\left(\operatorname{span}\left(\nabla \widetilde{Q}_{1}(x), \ldots, \nabla \widetilde{Q}_{k}(x)\right)\right)^{\perp}, \\
\Psi_{3}(x,(H, L, c), u) & =\nabla \widetilde{P}(x), \\
\Psi_{4}(x,(H, L, c), u) & =\widetilde{G}(x) u-\left(\left\langle\nabla \widetilde{Q}_{i}(x), \nabla \widetilde{P}(x)\right\rangle\right)_{i=1, \ldots, k}, \\
\Psi_{5}(x,(H, L, c), u) & =\left(\widetilde{Q}_{i}(x)\right)_{i=1, \ldots, k}
\end{aligned}
$$


Lemma 5 The mapping $\Psi$ is a submersion.

Proof. The partial derivative $\partial \Psi_{1} / \partial H(x,(H, L, c), u)$ is the identity of $\mathrm{Sym}_{n}$, $\partial \Psi_{3} / \partial L_{0}(x,(H, L, c), u)$ is the identity of $\mathbb{R}^{n}, \partial \Psi_{4} / \partial u(x,(H, L, c), u)$ is an isomorphism on $\mathbb{R}^{k}$ since $\widetilde{G}(x)$ is invertible and $\partial \Psi_{5} / \partial c(x,(H, L, c), u)$ is the identity of $\mathbb{R}^{k}$. It suffices to prove that the partial derivative

$$
\frac{\partial \Psi_{2}}{\partial\left(L_{1}, \ldots, L_{k}\right)}(x,(H, L, c), u)
$$

is surjective. This can be checked rather easily considering the definition of $\Psi_{2}$ and using $\nabla \widetilde{Q}_{i}(x)=\nabla Q_{i}(x)+L_{i}$.

Lemma 6 There exists an open dense subset $\mathcal{S}$ of $\mathcal{R}$ such that, for every perturbation $(H, L, c) \in \mathcal{S}$, the semialgebraic set $\theta_{\widetilde{M}}(\widetilde{P})$ is of dimension 1 .

Proof. For $(H, L, c)$ in $\mathcal{R}$, set

$$
\Psi_{H, L, c}: W \times \mathbb{R}^{k} \longrightarrow \operatorname{Sym}_{n} \times \mathbb{G}_{n-k, n} \times \mathbb{R}^{n} \times \mathbb{R}^{k} \times \mathbb{R}^{k}
$$

to be the mapping defined by $\Psi_{H, L, c}(x, u)=\Psi(x,(H, L, c), u)$. Note that $\theta_{\widetilde{M}}(\widetilde{P})$ is the projection of $\Psi_{H, L, c}^{-1}(\Sigma \times\{0\} \times\{0\})$ on $W$.

Lemma 5 allows us to apply the transversality theorem with parameters [13], giving an open dense subset $\mathcal{S}$ of $\mathcal{R}$ such that $\Psi_{H, L, c}$ is transverse to a finite semialgebraic stratification of $(\Sigma \times\{0\} \times\{0\}$ for every $(H, L, c)$ in $\mathcal{S}$. By Lemma 4. it follows that $\Psi_{H, L, c}^{-1}(\Sigma \times\{0\} \times\{0\})$ is of codimension $n-k-1+k+k=n+k-1$ in $W \times \mathbb{R}^{k}$, that is to say of dimension 1 . Hence, its projection $\theta_{\widetilde{M}}(\widetilde{P})$ is of dimension 1 .

Lemma 6] completes the proof of Proposition 2, Remark that the fact that the $Q_{i}$ are quadratic polynomials played no role in the proof. Hence, Proposition 2 actually holds for polynomials $Q_{1}, \ldots, Q_{k}$ of any degree $\geq 2$ and for any quadratic Morse function $P$ satisfying conditions (i) and (ii): up to an arbitrary small perturbation, one can assume that $\theta_{M}(P)$ is of dimension 1 .

We now assume that $\theta_{M}(P)$ is of dimension 1 . We can compute its length using Cauchy-Crofton formula, as the integral, on the Grassmannian $\mathbb{G}_{n-1, n}^{\text {aff }}$ of affine hyperplanes $h$ in $\mathbb{R}^{n}$ (w.r.t. a conveniently normalized measure $\mu$ ), of the number of intersection points $\sharp\left(h \cap \theta_{M}(P)\right)$ :

$$
\operatorname{length}\left(\theta_{M}(P)\right)=\int_{\mathbb{G}_{n-1, n}^{\text {aff }}} \sharp\left(h \cap \theta_{M}(P)\right) d \mu(h) .
$$

If we have a bound $I$ on the number of intersection points (for almost all $h$ ), then we obtain

$$
\operatorname{length}\left(\theta_{M}(P)\right) \leq I \nu(n),
$$

where $\nu(n)=2 \Gamma\left(\frac{1}{2}\right) \Gamma\left(\frac{n+1}{2}\right) \Gamma\left(\frac{n}{2}\right)^{-1}$ is the $\mu$-volume of the set of affine hyperplanes having a non-empty intersection with the unit ball.

The bound $I$ on the number of intersection points can be evaluated since $\theta_{M}(P)$ is a semialgebraic set, whereas the trajectories of the normalized gradient are not. Instead of applying directly Bézout's theorem to estimate the number of points in $h \cap \theta_{M}(P)$ as done in [10], which would give a bound exponential in $n$, we are going to use the methods introduced by Barvinok [2] in order to exploit the fact that we work with quadratic polynomials. 


\subsection{Barvinok's way: linear systems of small corank}

We proceed to prove:

Proposition 7 There is an open dense subset $\mathcal{V}$ of $\mathcal{U} \subset\left(\mathbb{R}_{2}\left[X_{1}, \ldots, X_{n}\right]\right)^{k+1}$ and a polynomial $p_{k}(n)$ of degree $O(k)$ such that, for every $\left(P, Q_{1}, \ldots, Q_{k}\right) \in \mathcal{V}$ and every affine hyperplane $h$ in $\mathbb{R}^{n}$, the number of connected components of $h \cap \theta_{M}(P)$ is $\leq p_{k}(n)$.

The intersection $h \cap \theta_{M}(P)$ is the set of $x \in \bar{B}^{n}$ such that there exist $\lambda \in \mathbb{R}$ and $\mu={ }^{t}\left(\mu_{1}, \ldots, \mu_{k}\right) \in \mathbb{R}^{k}$ satisfying the following system of equations:

$$
\begin{aligned}
\nabla\left(\left\|\nabla_{M} P\right\|^{2}\right)(x) & =\lambda \nabla P(x)+\sum_{i=1}^{k} \mu_{i} \nabla Q_{i}(x), \\
Q_{i}(x) & =0 \quad(i=1, \ldots, k), \\
{ }^{t} a x & =b,
\end{aligned}
$$

where the last equation is the equation of the hyperplane $h$.

We rewrite the quadratic polynomials $P(x), Q_{1}(x), \ldots, Q_{k}(x)$ as

$$
\begin{aligned}
P(x) & =\frac{1}{2}{ }^{t} x H_{0} x+{ }^{t} L_{0} x \\
Q_{i}(x) & =\frac{1}{2}{ }^{t} x H_{i} x+{ }^{t} L_{i} x+c_{i} \quad(i=1, \ldots, k),
\end{aligned}
$$

where $H_{i} \in \operatorname{Sym}_{n}, L_{i} \in \mathbb{R}^{n}$ and $c_{i} \in \mathbb{R}$. Then equation (7) becomes

$$
\begin{array}{r}
2\left(H_{0}-\sum_{i=1}^{k} u_{i}(x) H_{i}\right)\left(H_{0} x+L_{0}-\sum_{i=1}^{k} u_{i}(x)\left(H_{i} x+L_{i}\right)\right) \\
=\lambda\left(H_{0} x+L_{0}\right)+\sum_{i=1}^{k} \mu_{i}\left(H_{i} x+L_{i}\right) .
\end{array}
$$

Equation (10) fails to be a linear system in $x$ because of the $u_{i}(x)$. In order to make it linear in $x$, we introduce $k$ new variables $u={ }^{t}\left(u_{1}, \ldots, u_{k}\right) \in \mathbb{R}^{k}$ and add the $k$ equations (2) which relate $u$ and $x$. So finally $h \cap \theta_{M}(P)$ is described as the projection on $\mathbb{R}^{n}$ of the set of solutions $(x, \lambda, \mu, u) \in \mathbb{R}^{n} \times \mathbb{R} \times \mathbb{R}^{k} \times \mathbb{R}^{k}$ of a system of $n+2 k+1$ equations where the first $n$ are the following linear system in $x$ :

$$
\left[2 S(u)^{2}-\lambda H_{0}-\sum_{i=1}^{k} \mu_{i} H_{i}\right] x=S(u)\left(\sum_{i=1}^{k} u_{i} L_{i}-L_{0}\right)+\lambda L_{0}+\sum_{i=1}^{k} \mu_{i} L_{i}
$$

where $S(u)$ is the symmetric matrix $\left(H_{0}-\sum_{i=1}^{k} u_{i} H_{i}\right)$. The matrix of the system (11) depends on the parameters $(\lambda, \mu, u)$.

The main idea in Barvinok's paper is to solve the linear system for the (many) variables $x$ as rational functions of the parameters and possibly (few) free variables among $x$, using Cramer's formula. Substituting these expressions for $x$ in the remaining non linear equations reduces the total system to a system 
with few equations and few variables. The number of free variables among $x$ is equal to the corank of the matrix. Hence, following Barvinok, our first aim is to show that, for generic $\left(P, Q_{1}, \ldots, Q_{k}\right)$, the corank of this matrix is small for all parameters $(\lambda, \mu, u)$. However, the situation is more complicated here than in the paper [2], because of the presence of $S(u)^{2}$. set

For $(\lambda, \mu, u) \in \mathbb{R} \times \mathbb{R}^{k} \times \mathbb{R}^{k}=E$ and $H=\left(H_{0}, H_{1}, \ldots, H_{k}\right) \in\left(\mathrm{Sym}_{n}\right)^{k+1}$,

$$
\Phi(\lambda, \mu, u, H)=2\left(H_{0}-\sum_{i=1}^{k} u_{i} H_{i}\right)^{2}-\lambda H_{0}-\sum_{i=1}^{k} \mu_{i} H_{i}
$$

Proposition 8 There exists $c(k)=O(\sqrt{k}) \in \mathbb{N}$ and a dense open subset $\Omega \subset$ $\left(\operatorname{Sym}_{n}\right)^{k+1}$ such that, for every $H \in \Omega$ and every $(\lambda, \mu, u) \in E$, the corank of the matrix $\Phi(\lambda, \mu, u, H)$ is bounded by $c(k)$.

We cannot assume that the mapping $\Phi: E \times\left(\mathrm{Sym}_{n}\right)^{k+1} \rightarrow \mathrm{Sym}_{n}$ is submersive. But we can get close to that.

Lemma 9 There exists an open dense subset $\Omega^{\prime} \subset\left(\mathrm{Sym}_{n}\right)^{k+1}$ such that, for every $H \in \Omega^{\prime}$ and every $(\lambda, \mu, u) \in E$, the corank of the differential $d_{(\lambda, \mu, u, H)} \Phi$ is bounded by $k+1$.

Proof. We consider the partial derivative $\frac{\partial \Phi}{\partial H_{0}}(\lambda, \mu, u, H): \operatorname{Sym}_{n} \rightarrow \operatorname{Sym}_{n}$. Introducing the symmetric matrix $A(\lambda, u, H)=2\left(H_{0}-\sum_{i=1}^{k} u_{i} H_{i}\right)-\frac{\lambda}{2} I_{n}$ (where $I_{n}$ is the identity matrix), we obtain

$$
\frac{\partial \Phi}{\partial H_{0}}(\lambda, \mu, u, H): S \mapsto S A(\lambda, u, H)+A(\lambda, u, H) S .
$$

Denote by $m_{A}$ the endomorphism $S \mapsto S A+A S$ of $\operatorname{Sym}_{n}$. We use the following result.

Lemma 10 The set of $A \in \mathrm{Sym}_{n}$ such that the corank of $m_{A}$ is $\geq d$ has codimension $\geq d$ in $\mathrm{Sym}_{n}$.

Proof. If $a_{1}, \ldots, a_{n}$ are the eigenvalues of $A$, then the eigenvalues of $m_{A}$ are $a_{i}+a_{j}$ for $1 \leq i \leq j \leq n$ (this can easily be checked by reduction to the case when $A$ is diagonal). Hence the corank of $m_{A}$ is the number of sums of eigenvalues $a_{i}+a_{j}$ equal to 0 .

Consider diagonal matrices of the type

$$
D=\operatorname{diag}(\underbrace{0, \ldots, 0}_{\alpha}, \underbrace{b_{1}, \ldots, b_{1}}_{\beta_{1}}, \underbrace{-b_{1}, \ldots,-b_{1}}_{\gamma_{1}}, \ldots, \underbrace{b_{\ell}, \ldots, b_{\ell}}_{\beta_{\ell}}, \underbrace{-b_{\ell}, \ldots,-b_{\ell}}_{\gamma_{\ell}}),
$$

where $0<b_{1}<\ldots<b_{\ell}$, and $\alpha, \beta_{i}, \gamma_{i}$ are fixed nonnegative integers which sum up to $n$ and such that $\beta_{i}+\gamma_{i}>0$. They form a semialgebraic set of dimension $\ell$. The orbit of such a $D$ for the action by conjugation of the orthogonal group has dimension $o=\left(n(n-1)-\alpha(\alpha-1)-\sum_{i=1}^{\ell}\left(\beta_{i}\left(\beta_{i}-1\right)+\gamma_{i}\left(\gamma_{i}-1\right)\right)\right) / 2$. A simple computation shows that the codimension of the set of symmetric matrices similar to a matrix of type $D$, which is $n(n+1) / 2-o-\ell$, is greater than or equal to the corank of $m_{D}$, which is $\alpha(\alpha-1) / 2+\sum_{i=1}^{\ell} \beta_{i} \gamma_{i}$. Lemma 10] follows. 
We return to the proof of Lemma 9. The mapping

$$
(\lambda, u, H) \longmapsto A(\lambda, u, H)=2\left(H_{0}-\sum_{i=1}^{k} u_{i} H_{i}\right)-\frac{\lambda}{2} I_{n}
$$

is clearly submersive. Hence, by the theorem of transversality with parameters [13, there is an open dense subset $\Omega^{\prime} \subset\left(\operatorname{Sym}_{n}\right)^{k+1}$ such that, for every $H \in \Omega^{\prime}$, the mapping $(\lambda, u) \mapsto A(\lambda, u, H)$ from $\mathbb{R} \times \mathbb{R}^{k}$ to $\operatorname{Sym}_{\mathrm{n}}$ is transverse to a finite semialgebraic stratification of the subset of symmetric matrices $A$ such that $m_{A}$ has corank $>k+1$. Since this subset has codimension $>k+1$ by Lemma 10. this means that it has empty intersection with the image of $(\lambda, u) \mapsto A(\lambda, u, H)$. Thus the proof of Lemma 9 is completed.

In order to prove Proposition 8, we shall use a generalized transversality lemma which has a very simple proof in the semialgebraic context.

Lemma 11 Let $Z \subset \mathbb{R}^{m}$ and $F \subset \mathbb{R}^{n}$ be semialgebraic manifolds and $f: Z \rightarrow$ $F$, a smooth semialgebraic mapping such that the corank of $d_{x} f i s \leq c$ for every $x \in Z$. Let $B$ be a semialgebraic subset of codimension $b$ of $F$. Then the codimension of $f^{-1}(B)$ in $Z$ is $\geq b-c$.

Proof. Replacing $f$ with its graph, we may assume $Z \subset \mathbb{R}^{n+p}$ and $f$ is the restriction to $Z$ of the projection $\mathbb{R}^{n+p} \rightarrow \mathbb{R}^{n}$. Taking a cylindrical algebraic decomposition adapted to $F, Z$ and $B$ ([7, Section 5.1] or [8, Section 2.3]) we obtain that $F$ (resp. $Z$ ) is the disjoint union of a finite number of cells $S_{i}$ (resp. $T_{j}$ ) each diffeomorphic to $(0,1)^{d_{i}}$ (resp. $(0,1)^{e_{j}}$ ) and, for each $S_{i}$ there is a $T_{j}$ such that $f\left(S_{i}\right)=T_{j}$ and $\left.f\right|_{S_{i}}$ correspond via the diffeomorphisms to the projection of $(0,1)^{d_{i}}$ on the first $e_{j}$ coordinates. We have, for $x \in S_{i}$,

$$
d_{i}-e_{j} \leq \operatorname{dim}\left(\operatorname{ker}\left(d_{x} f\right) \leq \operatorname{dim} Z-\operatorname{dim} F+c\right.
$$

hence $\operatorname{codim}_{Z} S_{i} \geq \operatorname{codim}_{F} T_{j}-c$. Since $f^{-1}(B)$ is the union of those $S_{i}$ such that $T_{j}=f\left(S_{i}\right) \subset B$, the conclusion of the lemma follows.

We can now complete the

Proof of Proposition 8. It is known that the semialgebraic subset $B(c)$ of symmetric matrices of corank $\geq c$ is of codimension $c(c+1) / 2$ in $\operatorname{Sym}_{n}$ (cf. for instance corollary to Lemma 2 in [1). Using Lemmas 9 and 11, we obtain $\Omega^{\prime}$ open and dense in $\left(\operatorname{Sym}_{n}\right)^{k+1}$ such that the codimension of $\Phi^{-1}(B(c))$ in $E \times \Omega^{\prime}$ is $\geq c(c+1) / 2-(k+1)$. Hence, the projection $p_{\Omega^{\prime}}\left(\Phi^{-1}(B(c))\right)$ has codimension $\geq c(c+1) / 2-(3 k+2)$ in $\Omega^{\prime}$. If we take $c$ such that $c(c+1) / 2>3 k+2$, for instance

$$
c=c(k)=\left\lfloor\frac{1+\sqrt{17+24 k}}{2}\right\rfloor,
$$

the complement of $p_{\Omega^{\prime}}\left(\Phi^{-1}(B(c))\right)$ in $\Omega^{\prime}$ contain a dense open subset $\Omega$ such that, for every $H \in \Omega$ and every $(\lambda, \mu, u) \in E$, the corank of $\Phi(\lambda, \mu, u, H)$ is $<c$.

Once we have this result, we follow closely Barvinok in the 
Proof of Proposition 7, Let $\mathcal{V} \subset \mathcal{U}$ be the open dense subset of $\left(P, Q_{1}, \ldots, Q_{k}\right)$ such that the corresponding $H$ belongs to $\Omega$ as in Proposition 8 . Assume in the following that $\left(P, Q_{1}, \ldots, Q_{k}\right) \in \mathcal{V}$.

The intersection $h \cap \theta_{M}(P)$ is the projection on the space $\mathbb{R}^{n}$ of variables $x$ of the set of solutions of the following system of $n+2 k+1$ equations in the $n+2 k+1$ variables $x, \lambda, \mu, u$

$$
\begin{aligned}
\Phi x & =C, \\
Q_{i}(x) & =0 \quad(i=1, \ldots, k), \\
G(x) u & =\left(\left\langle\nabla Q_{i}(x), \nabla P(x)\right\rangle\right)_{i=1, \ldots, k}, \\
{ }^{t} a x & =b,
\end{aligned}
$$

where $\Phi=\Phi(\lambda, \mu, u, H)$ and

$$
C=\left(H_{0}-\sum_{i=1}^{k} u_{i} H_{i}\right)\left(\sum_{i=1}^{k} u_{i} L_{i}-L_{0}\right)+\lambda L_{0}+\sum_{i=1}^{k} \mu_{i} L_{i} .
$$

We denote by $\widehat{\Phi}$ the augmented matrix $(\Phi, C)$. The coefficients of $\widehat{\Phi}$ are polynomials in $\lambda, \mu, u$ of total degree $\leq 2$. The equations (15) and (16) are quadratic in $x$.

First we solve the linear system (14) in $x$ over pieces of the space $E$ of parameters $(\lambda, \mu, u)$ where a uniform choice of free variables among $x$ can be made. Let $\delta=(s, I, J)$ where $s$ is a nonnegative integer $\leq c(k)(c(k)$ as in Proposition (8) and $I$ and $J$ are subsets of $\{1, \ldots, n\}$ of cardinal $s$. Denote by $\Phi_{I, J}$ the $(n-s) \times(n-s)$ submatrix of $\Phi$ obtained by deleting all rows with index in $I$ and all columns with index in $J$. Set

$$
B_{\delta}=\left\{(\lambda, \mu, u) \in E \mid \operatorname{det}\left(\Phi_{I, J}\right) \neq 0 \text { and } \operatorname{rank}(\widehat{\Phi})=n-s\right\} .
$$

The semialgebraic set $B_{\delta}$ is described by an inequality $\left(\operatorname{det}\left(\Phi_{I, J}\right)^{2}>0\right)$ and an equation (expressing that the sum of the squares of all $(n-s+1) \times(n-s+1)$ minors extracted from $\widehat{\Phi}$ is zero) in $(\lambda, \mu, u)$, both of total degree $\leq 4 n$. The total number of $B_{\delta}$ 's is $\sum_{s=0}^{c(k)}\left(\begin{array}{l}n \\ s\end{array}\right)^{2} \leq(c(k)+1) n^{2 c(k)}$.

Fix a $\delta=(s, I, J)$. For every $(\lambda, \mu, u) \in B_{\delta}$, we can choose $x_{J}=\left(x_{j}\right)_{j \in J}$ as free variables and describe, using Cramer's formula, the solutions of the linear system (14) as $x\left(\lambda, \mu, u, x_{J}\right)$ where the coordinates are rational functions of total degree $\leq 2 n+1$ with a common denominator. Substituting this solution for $x$ in the remaining equations (15, 16, 17) and adding the equation and the inequality describing $B_{\delta}$, we get a system $\Sigma_{\delta}$ of $2 k+2$ equations and one inequality in $\leq 2 k+1+c(k)$ unknowns $\lambda, \mu, u, x_{J}$ of total degree $\leq 4 n+3$. The number of connected components of the set of solutions of $\Sigma_{\delta}$ is bounded from above by $(4 n+3)(8 n+5)^{2 k+1+c(k)}$ (see for instance the bound in [8, Proposition 4.13] or more precise bounds in [7]).

Since $h \cap \theta_{M}(P)$ is covered by the images of the sets of solutions of $\Sigma_{\delta}$ 's by the mappings $\left(\lambda, \mu, u, x_{J}\right) \mapsto x\left(\lambda, \mu, u, x_{J}\right)$, the number of connected components of $h \cap \theta_{M}(P)$ is bounded from above by

$$
p_{k}(n)=(c(k)+1) n^{2 c(k)}(4 n+3)(8 n+5)^{2 k+1+c(k)} .
$$




\subsection{Putting all together}

Theorem 12 Let $M$ be the intersection of the closed unit ball $\bar{B}^{n} \subset \mathbb{R}^{n}$ with the intersection $X$ of $k$ quadrics $Q_{i}(x)=0$, such that $M$ is a submanifold without boundary of $B^{n}$ and a complete intersection of codimension $k$. Then the sum of the geodesic diameters of the connected components of $M$ is bounded from above by a polynomial $q_{k}(n)$ of of degree $O(k)$.

Proof. Choose a quadratic Morse function $P$ on $M$ and assume $\left(P, Q_{1}, \ldots, Q_{k}\right)$ is in the intersection of $\mathcal{U}^{\prime}$ of Proposition 2 and $\mathcal{V}$ of Proposition 7 Then $\theta_{M}(P)$ is a semialgebraic set of dimension 1 , and twice its length bounds from above the sum of the geodesic diameters of the connected components of $M$. By Proposition 7 and Cauchy-Crofton formula, the length of $\theta_{M}(P)$ is bounded from above by $\nu(n) p_{k}(n)$, where $\nu(n)=2 \Gamma\left(\frac{1}{2}\right) \Gamma\left(\frac{n+1}{2}\right) \Gamma\left(\frac{n}{2}\right)^{-1} \leq \sqrt{\pi} n$. Hence the sum of the geodesic diameters of the connected components of $M$ is bounded from above by $q_{k}(n)=2 \sqrt{\pi} n p_{k}(n)$, a polynomial in $n$ of degree $O(k)$.

Every $\left(P, Q_{1}, \ldots, Q_{n}\right)$ in $\mathcal{U}$ can be approximated by $\left(\widetilde{P}, \widetilde{Q}_{1}, \ldots, \widetilde{Q}_{n}\right)$ in the open dense subset $\mathcal{U}^{\prime} \cap \mathcal{V}$. Hence every $M$ as in the theorem can be approximated in the Hausdorff topology by $\widetilde{M}$ for which the bound on the sum of geodesic diameters holds. From this follows that the bound also holds for $M$ (cf. [10, Lemma 6.2]).

\section{Semialgebraic sets defined by quadratic inequal- ities}

We now extend step by step the result obtained for smooth complete intersection of quadrics to the general case of semialgebraic sets defined by quadratic equations and inequalities.

Let $Q_{1}, \ldots, Q_{k}$ be quadratic polynomials in $n$ variables. Set $Q_{0}(x)=1-$ $\sum_{i=1}^{n} x_{i}^{2}$. We first consider the case

$$
M=\left\{x \in \mathbb{R}^{n} \mid Q_{0}(x) \geq 0, Q_{1}(x) \geq 0, \ldots, Q_{k}(x) \geq 0\right\} \subset \bar{B}^{n},
$$

where we assume moreover that the $Q_{i}(x)=0$ for $i=0, \ldots, k$ are normal crossing divisors inside $\bar{B}^{n}$. That is, for all $\left\{i_{1} \ldots, i_{\ell}\right\} \subset\{0, \ldots, k\}$ and all $x \in \bar{B}^{n}$ such that $Q_{i_{1}}(x)=\ldots=Q_{i_{\ell}}(x)=0$, then $\nabla Q_{i_{1}}(x), \ldots, \nabla Q_{i_{\ell}}(x)$ are linearly independent.

Proposition 13 With the assumption above, the sum of the geodesic diameters of the connected components of $M$ is bounded by $r_{k}(n)$, a polynomial in $n$ of degree $O(k)$.

Proof. Let $y=\left(y_{0}, \ldots, y_{k}\right)$ be new variables and $a_{0}, \ldots, a_{k}$ real numbers such that

$$
0<a_{i}<\frac{1}{(k+1) \max _{x \in \bar{B}^{n}}\left(\left|Q_{i}(x)\right|\right)} .
$$

Set

$$
\widehat{M}=\left\{(x, y) \in \mathbb{R}^{n} \times \mathbb{R}^{k+1} \mid y_{i}^{2}-a_{i} Q_{i}(x)=0, i=0, \ldots, k\right\} .
$$

If $(x, y) \in \widehat{M}$, then $\|x\|^{2} \leq 1$ and $y_{i}^{2} \leq 1 /(k+1)$, so $\widehat{M}$ is contained in the open ball $2 B^{n}$ of radius 2 . Moreover, due to the assumption on normal crossings, $\widehat{M}$ 
is the smooth complete intersection of $k+1$ quadrics in $\mathbb{R}^{n+k+1}$. Hence we can apply Theorem 12 (after a homothecy with ratio $1 / 2$ ) and obtain that the sum of the geodesic diameters of the connected components of $\widehat{M}$ is bounded from above by $r_{k}(n)=2 q_{k+1}(n+k+1)$, a polynomial in $n$ of degree $O(k)$.

Since $M$ is the image of $\widehat{M}$ by the projection $(x, y) \mapsto x$ which does not increase the lengths and induces a bijection between connected components of $\widehat{M}$ and connected components of $M$, the same bound holds for the sum of the geodesic diameters of the connected components of $M$.

Next we turn to the case of an intersection of quadrics, without any assumption.

Proposition 14 Let $X=\left\{x \in \mathbb{R}^{n} \mid Q_{1}(x)=\ldots=Q_{k}(x)=0\right\}$ be any intersection of $k$ quadrics in $\mathbb{R}^{n}$. Let $M$ be the intersection of $X$ with the closed unit ball $\bar{B}^{n}$. Then the sum of the geodesic diameters of the connected components of $M$ is bounded from above by $s_{k}(n)$, a polynomial in $n$ of degree $O(k)$.

Proof. For $\epsilon=\left(\epsilon_{1}, \ldots, \epsilon_{k}\right)$ with $\epsilon_{i}>0$, set

$$
M_{\epsilon}=\left\{x \in \bar{B}^{n} \mid-\epsilon_{i} \leq Q_{i}(x) \leq \epsilon_{i}, i=1, \ldots, k\right\} .
$$

The semialgebraic Sard theorem [7. Theorem 5.57] imply that there is an open dense subset $\mathcal{W}$ of $\left\{\epsilon \in \mathbb{R}^{k} \mid \epsilon_{i}>0, i=1, \ldots, k\right\}$ such that, for all $\left\{i_{1}, \ldots, i_{\ell}\right\} \subset$ $\{1, \ldots, n\}$ and all $\left(\delta_{1}, \ldots, \delta_{\ell}\right) \in\{-1,1\}^{\ell},\left(\delta_{1} \epsilon_{i_{1}}, \ldots, \delta_{\ell} \epsilon_{i_{\ell}}\right)$ is a regular value of $\left(Q_{i_{1}}, \ldots, Q_{i_{\ell}}\right)$ and of its restriction to the unit sphere $S^{n-1}$. For $\epsilon \in \mathcal{W}, M_{\epsilon}$ falls in the case of inequalities with normal crossings, and by Proposition 13 the sum of the geodesic diameters of the connected components of $M_{\epsilon}$ is bounded from above by $s_{k}(n)=r_{2 k}(n)$, a polynomial in $n$ of degree $O(k)$.

Since $M$ is the limit for Hausdorff topology of $M_{\epsilon}$ 's with $\epsilon \in \mathcal{W}$, the same bound holds for the sum of the geodesic diameters of the connected components of $M$ (cf. [10, Lemma 6.2]).

Finally we arrive to the general situation.

Theorem 15 Let $Q_{1}, \ldots, Q_{k}$ be quadratic polynomials in $n$ variables and let

$$
\begin{array}{r}
X=\left\{x \in \mathbb{R}^{n} \mid Q_{1}(x)=\ldots=Q_{\ell}(x)=0, Q_{\ell+1} \geq 0, \ldots, Q_{m}(x) \geq 0\right. \\
\left.Q_{m+1}(x)>0, \ldots, Q_{k}(x)>0\right\} .
\end{array}
$$

Let $M$ be the intersection of $X$ with the closed unit ball $\bar{B}^{n}$. Then the sum of the geodesic diameters of the connected components of $M$ is bounded from above by $t_{k}(n)$, a polynomial in $n$ of degree $O(k)$.

Proof. Assume first there is no strict inequality in the description of $X$. Then we can treat the nonstrict inequalities as in the proof of Proposition 13 to reduce to an intersection of quadrics and apply Proposition [14. In this way we obtain a polynomial bound $t_{k}(n)$ of degree $O(k)$ for the sum of the geodesic diameters of the connected components of $M$.

Consider now $X$ as in the statement of the proposition and assume that the sum of the geodesic diameters of the connected components $M_{j}(j=1, \ldots, p)$ 
of $M$ is $>t_{k}(n)$. Pick a couple of points $\left(x_{j}, y_{j}\right)$ in each $M_{j}$ such that the sum of the geodesic distances from $x_{j}$ to $y_{j}$ in $M_{j}$ is $>t_{k}(n)$. Choose a path $\gamma_{j}$ from $x_{j}$ to $y_{j}$ in $M_{j}$ and set $\epsilon=\min _{i=m+1, \ldots, k, j=1, \ldots, p, t \in[0,1]}\left(Q_{i}\left(\gamma_{j}(t)\right)\right.$. Let $M_{\epsilon} \subset M$ be obtained by replacing $Q_{i}(x)>0$ with $Q_{i}(x) \geq \epsilon$ for $i=m+1, \ldots, k$. Then $x_{j}$ and $y_{j}$ are in the same connected of $M_{\epsilon}$ and their geodesic distance in this connected component is at least equal to their geodesic distance in $M_{j}$. This contradicts the fact, established above, that the sum of the geodesic diameters of the connected components of $M_{\epsilon}$ is at most $t_{k}(n)$.

\section{Questions}

One would get a much simpler proof for the crucial case of the smooth intersection of quadrics (and also a better bound) if one could consider only the case when the linear system (14) in $x$ is always of maximal rank. This would be all right if the assumption of maximal rank only removes finitely many points from the one-dimensional set $\theta_{M}(P)$ and does not affect the computation of its length. Unfortunately, we have not been able to push this idea.

In the case when there is only one quadratic inequality, then the number of connected components of $\bar{B}^{n} \cap\left\{x \in \mathbb{R}^{n} \mid Q(x) \geq 0\right\}$ is at most two, and the geodesic diameter of a connected component of $\bar{B}^{n} \cap\left\{x \in \mathbb{R}^{n} \mid Q(x) \geq 0\right\}$ is at most $2 \pi$, independently of $n[15]$.

So the first case when the question of the dependence on $n$ of the sum of geodesic diameters is relevant is the case of two quadratic inequalities (actually there are altogether three quadratic inequalities since we intersect with the unit ball). What is the optimal exponent of $n$ for a bound on the sum of the geodesic diameters of the connected components of $\bar{B}^{n} \cap\left\{x \in \mathbb{R}^{n} \mid Q_{1}(x) \geq 0, Q_{2}(x) \geq\right.$ $0\}$ ?

A similar problem for the maximal number of connected components of an intersection of three quadrics has been recently solved by Degtyarev, Kharlamov and Itenberg [11]: they obtain lower and upper bounds in $n^{2}$. However their very nice proof relies on rather sophisticated arguments and gives no insight on metric properties. The question remains of constructing families of quadratic polynomials $Q_{1, n}, Q_{2, n}$ in $n$ variables such that the sum of the geodesic diameter of $\bar{B}^{n} \cap\left\{x \in \mathbb{R}^{n} \mid Q_{1, n}(x) \geq 0, Q_{2, n}(x) \geq 0\right\}$ tends to infinity with $n$.

\section{References}

[1] A.A. Agrachev: The topology of quadratic mappings and Hessians of smooth mappings, J. Soviet Math. 49: 990-1013 (1990)

[2] A.I. Barvinok: On the Betti numbers of semialgebraic sets defined by few quadratic inequalities, Math. Zeit. 225: 231-244 (1997)

[3] S. Basu: Computing the top Betti numbers of semi-algebraic sets defined by quadratic inequalities in polynomial time, Found. Comput. Math. 8: 45-80 (2008)

[4] S. Basu, M. Kettner: A sharper estimate on the Betti numbers of sets defined by quadratic inequalities, Discrete Comput. Geom. 39: 734-746 (2008) 
[5] S. Basu, D. Pasechnik, M-F. Roy: Computing the Betti numbers and the Euler-Poincare characteristic of semi-algebraic sets defined by partly quadratic systems of polynomials, J. Algebra 321: 2206-2209 (2009)

[6] S. Basu, D. Pasechnik, M-F. Roy: Bounding the Betti numbers of semialgebraic sets defined by partly quadratic systems of polynomials, J. Eur. Math. Soc. 12: 529-553 (2010)

[7] S. Basu, R. Pollack, M-F. Roy: Algorithms in real algebraic geometry. Springer Verlag (2006) http://perso.univ-rennes1.fr/marie-francoise.roy/bpr-ed2-posted1.pdf

[8] M. Coste: An introduction to semialgebraic geometry (2002). http://perso.univ-rennes1.fr/michel.coste/polyens/SAG.pdf

[9] D. D'Acunto, K. Kurdyka: Bounds for gradient trajectories of polynomial and definable functions with applications. Preprint (2004) http://www.lama.univ-savoie.fr/ d_acunto/dacunto-kurdyka1.pdf

[10] D. D'Acunto, K. Kurdyka: Bounds for gradient trajectories and geodesic diameter of real algebraic sets, Bull. London Math. Soc. 38: 951-965 (2006)

[11] A. Degtyarev, I. Itenberg, V. Kharlamov: On the number of components of a complete intersection of real quadrics. arXiv:0806.4077

[12] D. Grigoriev, D. Pasechnik: Polynomial-time computing over quadratic maps. I. Sampling in real algebraic sets. Comput. Complexity 14: 20-52 (2005)

[13] V. Guillemin, A. Pollack: Differential topology. Prentice Hall (1974)

[14] S. Moussa: Bornes pour le diamètre géodésique d'une composante connexe d'un ouvert semi-algébrique, Bull. Sci. Math. 132: 70-77 (2008)

[15] S. Moussa: Aspects quantitatifs des ensembles semi-algébriques. Thesis UAM Niamey - Université Rennes 1 (2009)

Institut de Recherche MAthématique de Rennes

Université de Rennes 1 et CNRS

Campus de Beaulieu

35042 Rennes cedex, France

michel.coste@univ-rennes1.fr

Département de Mathématiques

Faculté des Sciences et Techniques

Université Abdou Moumouni

BP 10662 Niamey, Niger

seydmoussa@yahoo.fr 